\title{
Erratum
}

\section{A New Approach to Methoxyisatins Leading to the Total Synthesis of Ophiuroidine and Other Hydroxytryptanthrins}

Jeffrey J. Mason, Tomasz Janosik, Jan Bergman* Synthesis 2009, 3642.

The advance online publication (e-First) version of this article contained an incorrect phenyl ring substitution pattern for compounds 8-10 in Scheme 2.

This mistake has been corrected for both the current online version and the print publication. 